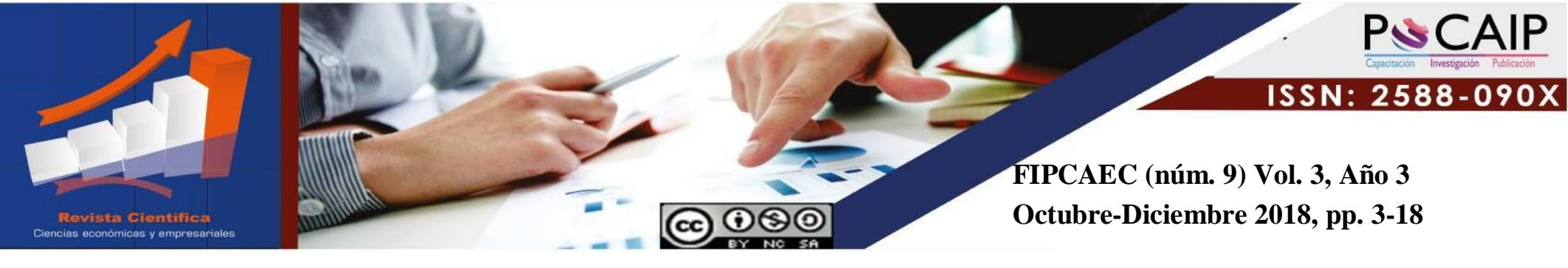

DOI: $10.23857 /$ fipcaec.v3i9.50

\title{
Empowerment como estrategia para la gerencia empresarial
}

\author{
Empowerment as a strategy for business management \\ O empoderamento como estratégia de gestão de negócios
}

\author{
Dayni Lisset Palacios-Molina ${ }^{1}$ \\ daynilisseth1905@yahoo.com \\ https://orcid.org/0000-0001-5358-1804
}

Correspondencia: daynilisseth1905@yahoo.com

* Recepción: 12/ 07/ 2018 * Aceptación: 14/09/ 2018 *Publicación: 15 /10/ 2018

Magíster en Administración de Empresas, Ingeniera Comercial Especialización Mayor Negocios Internacionales Especialización Menor Marketing y Ventas, Docente de la Universidad Laica Eloy Alfaro de Manabí, Facultad Ciencias Administrativas, Manta, Ecuador. 


\title{
Resumen
}

La investigación fue de tipo descriptiva con el objetivo de analizar el empowerment como estrategia para la gerencia empresarial de la cooperativa de transporte MTC de la provincia de Manabí - Ecuador con la finalidad de conocer cómo se desarrolla el empowerment en esta organización. Los resultados demuestran claramente que existe debilidades se infiere que los trabajadores tienen debilidades en cuanto al indicador solución de conflictos por lo tanto también debilidades en las relaciones interpersonales. El talento humano de la organización requiere proseguir en la formación del empowerment como estrategia de empoderamiento gerencial, de ese modo, existirá mayor posibilidad de que se genere un liderazgo comprometido en alcanzar el mayor nivel de productividad en conformidad de relación con la filosofía organizacional de la empresa.

Palabras clave: Cooperativa; cambio organizacional; modernización; comportamiento innovador.

\begin{abstract}
The research was descriptive in order to analyze the empowerment as a strategy for business management of the MTC transport cooperative in the province of Manabí - Ecuador in order to know how the empowerment develops in this organization. The results clearly show that there are weaknesses, it is inferred that workers have weaknesses in terms of the conflict resolution indicator, therefore also weaknesses in interpersonal relationships. The human talent of the organization requires continuing in the formation of empowerment as a strategy of managerial empowerment, in this way, there will be a greater possibility of generating a leadership committed to achieving the highest level of productivity in accordance with the organizational philosophy of the company.
\end{abstract}

keywords: Cooperatives; organizational change; modernization; innovation behaviour.

\section{Resumo}

A pesquisa foi descritiva, com o objetivo de analisar o empoderamento como estratégia para a 


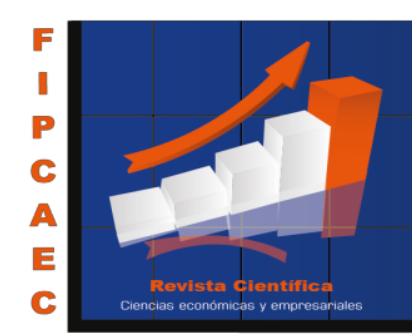

conhecer como o empoderamento se desenvolve nessa organização. Os resultados mostram claramente que existem fragilidades, infere-se que os trabalhadores apresentam fragilidades em termos do indicador de resolução de conflitos, portanto também fragilidades nas relações interpessoais. O talento humano da organização exige a continuidade da formação do empoderamento como estratégia de empoderamento gerencial, assim, haverá maior possibilidade de gerar uma liderança comprometida em alcançar o mais alto nível de produtividade, de acordo com a filosofia organizacional da organização. companhia

Palavras-chave: Cooperativa; mudança organizacional; modernização; comportamento inovador

\section{Introducción}

Las empresas desde la competividad global, han sumado como estrategia fundamental, la formación permanente de su talento humano, esto con la finalidad de estar a la altura de las exigencias del mercado en la medida que su cliente interno puede entenderlas, dado la diversidad tecnológica - mercadeo existente, se hace necesario que el talento humano se empodere de la filosofía de gestión organizacional, siendo así la generación de fidelidad, por lo consiguiente un enfoque generador de empoderamiento es el empowerment, el cual se basa en dar poder y autoridad a los empleados. Así los trabajadores tendrán influencia y poder de decisión sobre su trabajo sin necesidad de requerir constante permiso para actuar, esto no significa una pérdida de autoridad de la alta gerencia.

Siendo el empowerment una nueva forma de gestionar el talento humano, delegando autoridad y responsabilidad trayendo beneficios tales como: incremento de la creatividad, aumento de la responsabilidad, autoridad y compromiso en la satisfacción del cliente, además contribuye a impulsar el liderazgo participativo y el trabajo en equipo. En este sentido, Valdez (2005), describe el empowerment como:

La estrategia se apoya en la premisa de quienes están relacionados directamente con el puesto de trabajo son los más indicados para tomar las decisiones al respecto, ya que son quienes conocen y poseen las actitudes para ello, la participación de todos los trabajadores en el proceso de mejora continua permitirá a cualquier institución lograr el éxito (p. 58). 
Para fomentar esta participación es necesario facultar al personal para la toma de decisiones orientadas a proporcionar solución esa problemas; generando en los trabajadores una sensación de pertenencia y se desarrollando el espíritu de colaboración empresa-trabajador. Es interesante saber que muchas empresas a nivel mundial han mejorado en su funcionamiento, aplicando esta técnica y que los resultados han sido satisfactorios. Con esta estrategia se logra una nueva manera de administrar, una filosofía de manejar el talento humano de una organización para lograr sus objetivos.

De allí la importancia del empoderamiento como un proceso gerencial que permite la influencia mutua en la distribución creativa del poder y representa una responsabilidad compartida, logrando que las personas utilicen sus talentos y capacidades para una gestión del conocimiento más exitosa, por cuanto a través de una gerencia, específicamente educativa de calidad se utilizan las mejores prácticas para delegar responsabilidades, desarrollando así el capital humano.

Se puede afirmar entonces que el empoderamiento se convierte en una herramienta vital para la transformación del proceso gerencial de las instituciones educativas en la actualidad; por ello, la importancia de utilizar al máximo estrategias gerenciales basadas en este enfoque, el cual propicia en las organizaciones el fortalecimiento del personal directivo, logrando un cambio en la cultura organizacional como una cultura de apoyo, con equipos de trabajo auto dirigidos, orientada hacia el logro de metas y objetivos planteados para tal fin, el empoderamiento del talento humano con la organización permite elevar la calidad del servicio al cliente, Aldana \& Piña (2017).

En tal sentido, Cálix, Martínez, Vigier \& Núñez (2016), señalan que “el éxito empresarial se basa en tener objetivos estratégicos acertados y una cultura corporativa adecuada que acoja los valores de la empresa, el clima organizacional, el estilo gerencial y la aplicación de diversas herramientas administrativas” (p. 23), siendo esto posible desde la adopción del empowerment como estrategia gerencial empresarial, por cuanto permite aglutinar diversas áreas de la empresa para crear compromiso por parte del talento humano con la misma, partiendo de la importancia del empowerment en la mejora continua de la empresa, la actual investigación se focalizar en analizar el empowerment como estrategia para la gerencia empresarial de la cooperativa de 


\section{Características del Empowerment}

Desde el dinamismo gerencial planteado por el empowerment, Valdés (2005), destaca las siguientes características:

1. Orgullo: Apreciar el gusto por hacer las cosas bien continuamente.

2. Unión y solidaridad: mayor cohesión del grupo y por lo tanto un trabajo en equipo.

3. Voluntad: Disponibilidad para contribuir a las metas propuestas.

4. Atención a los detalles: Crear el hábito de no pasar por alto ningún detalle por pequeño que parezca especialmente en aquellos relacionados con la producción y el cliente.

5. Credibilidad: Cumplir las promesas realizadas inspirando la confianza de todos los miembros de la empresa.

Las características del empowerment son de gran relevancia debido a que, representa el orgullo que sienten las personas por realizar las actividades idóneamente y saber que éstas son significativas para la organización. La unidad es fundamental entre los miembros porque va a permitir la consecución de las metas planteadas y por ende ofrecer al cliente un servicio más eficaz y eficiente. La solidaridad genera responsabilidad por resultados de forma grupal dejando de lado el individualismo. Con empowerment se desarrolla la voluntad de las personas para contribuir a los fines de la empresa.

\section{Principios del Empowerment}

El Empowerment como el resto de todas las demás herramientas y técnicas tiene que estar fundamentado o basado en unos principios. Al respecto Johnson (2005, p. 4) plantea los siguientes principios:

1. Establecer autoridad y responsabilidad sobre las actividades.

2. Definir en conjunto modelos de excelencia.

3. Proveer de la retroalimentación oportuna sobre el desempeño de los miembros del proceso. 
6. El aumento de la motivación para colaborar, manifestándose una actitud positiva en todas las personas.

7. Se dinamiza los procesos para una toma de decisiones más oportuna y eficiente.

El empowerment es donde se manifiesta y se le saca provecho al potencial que posee cada persona que forma parte de la institución, es donde se palpa la creatividad, la capacidad innovadora de las personas, sus competencias, habilidades, conocimientos e inclusive sus debilidades y fortalezas. Empowerment es dar autonomía e independencia los trabajadores en sus tareas, es reconocer sus esfuerzos, es impulsarlos al crecimiento personal, es trabajar en equipo, es distribuir la comunicación por todos los niveles para que éstos sepan lo que realmente está pasando en la empresa como se están realizando las actividades y si realmente se están cumpliendo los objetivos planteados. El empowerment se basa en cuatro aspectos principales: poder, motivación, desarrollo y liderazgo. Cuando se les otorga poder a las personas se les está demostrando que se confía en ellas, que son importantes para la empresa, lo que crea un sentido de pertenencia en los trabajadores porque saben que la organización cuenta y necesita de su apoyo.

\section{Requisitos para la implementación de Empowerment}

Desde la perspectiva de Acosta (2002), se hace necesario contar con los siguientes requisitos que permitan la implementación efectiva del empowerment:

1. El primer requisito para implementar el empowerment es contar con el todo el apoyo de la alta gerencia de la empresa.

2. La organización deberá tener claramente definida una visión, una estrategia, unos valores, unas metas que deben ser conocidas y compartidas por todos los integrantes de la compañía. La visión establece lo que se quiere alcanzar, hacia donde debe ir la organización.

3. Creación de equipos de trabajo de una forma escalonada. 
Oportunidades de capacitación: hay que verificar si el equipo necesita orientación y de ser así brindársela.

Nueva tecnologías: para comenzar a trabajar debe haber un ambiente de trabajo ergonómico. Por lo tanto, es necesario contar con una buena tecnología, donde los empleados se sientan cómodos y motivados.

El personal: es bueno saber que pueden ofrecer los empleados, que les agrada y en qué son competentes, para ayudarlos a vincular sus capacidades con los objetivos que establece la organización.

Buscar ayuda: establecer el tipo de ayuda que se necesita para poner en marcha la herramienta, se deben identificar las dificultades. Es conveniente estar al tanto donde se pueden presentar y así poder prever soluciones.

Tener muy claro el punto de partida: Lo primero que se observa en la organización es su cultura organizacional, para conocer de qué manera se realizan las actividades en la misma, si la empresa es muy rígida y se resiste al cambio el proceso de facultación suele ser más complejo. Preparar las bases para la aplicación del empowerment significa que se deben conjugar todos los factores necesarios, lo que exige una revisión total de la forma en que esta técnica puede satisfacer las necesidades propias y organizacionales, y cómo se puede obtener.

\section{Barreras al facultar el Empowerment}

Debido a que facultar significa permitir a las personas asumir responsabilidades, es preciso pensar en las trabas que se puedan presentar y por eso es necesario crear un plan para abatir todas las barreras.

Hacer una tormenta de ideas personal: es situarse en lugar de los demás, es decir, indagar las limitantes de los empleados. Lo importante es explorar todos los rincones y recaudar toda la información necesaria.

Reunir al equipo y probarlo: consiste en reunir al grupo y descubrir cosas de ellos y ver cuáles son las barreras que creen enfrentar, no se deben descalificar o anular las sugerencias de ninguno, ni tratar de objetar las limitantes que se formulan. 


\section{Metodología de la investigación}

Se trabajó con una investigación tipo descriptiva con el objetivo de analizar el empowerment como estrategia para la gerencia empresarial de la cooperativa de transporte MTC de la provincia de Manabí - Ecuador con la finalidad de conocer cómo se desarrolla el empowerment en esta organización, ante lo cual, Sabino (2000) plantea que esta tipología de investigación se tiene "su preocupación primordial radica en describir algunas características fundamentales de conjunto homogéneos de fenómenos" (p. 62), complementándose en un diseño de campo no experimental.

En cuanto a la población, Sabino (2000) la define como, "la totalidad del fenómeno a estudiar, donde las unidades de población poseen características comunes, que se estudian y dan origen a los datos de la investigación” (p.204), se trabajó con una muestra poblacional de 35 personas que forman parte del talento humano de la empresa objeto de estudio, a quienes se les aplicó un instrumento de 25 alternativas de repuestas con escalamiento tipo Likert de cinco alternativas de respuestas, aplicándose el coeficiente Alfa de Cronbach de 0,89 catalogándose como altamente confiable para su aplicación.

\section{Análisis y discusión de los resultados}

Para el indicador habilidades de liderazgo, el $20 \%$ de los trabajadores, afirman que siempre considera que posee habilidades para llevar a cabo la gerencia educativa, mientras que el $40 \%$ considera que casi siempre, $20 \%$ algunas veces, $36 \%$ pocas veces y finalmente $21 \%$ nunca. En forma general, estos resultados permiten afirmar que el indicador habilidades tienen un comportamiento positivo, por lo tanto constituye una gran fortaleza y está en La dirección de lo planteado por Newstrom (1992), quien sostiene que la "habilidad es la capacidad de realizar una acción relacionada con el trabajo, es la combinación del conocimiento con destreza física y que se adquiere mediante la práctica" (p. 225).

Para el indicador conocimientos, el cual $20 \%$ consideran que casi siempre tienen los conocimientos necesarios para el desarrollo de sus funciones como gerente educativo, 40\% algunas veces, $20 \%$ pocas veces y $20 \%$ nunca, al realizar un balance de los resultados de los ítems 2, se determinó que existe una debilidad en cuanto al indicador conocimientos y habilidades, la aplicación del conocimiento es necesario en las organizaciones como lo afirma 
Chirinos (2009), el conocimiento es "el producto de nuestras experiencias, valores, de la información que obtenemos del entorno, de las evaluaciones y análisis que realizamos” (p. 27). Pero, si se parte de aceptar el surgimiento y aplicación del conocimiento está en la mente de las personas, las organizaciones no pueden perderlo de vista o conformarse con guardar parte de el en planes, formatos, bases de datos, normas, procedimientos, entre otros.

En cuanto al indicador toma de decisiones, el 20\% de los trabajadores opinaron que casi siempre toma decisiones con frecuencia en el desarrollo de sus funciones, 20\% algunas veces, $40 \%$ pocas veces y $20 \%$ nunca. Los aspectos antes descritos indican que el indicador toma de decisiones tiene una tendencia negativa, no se promueve en la empresa la toma de decisiones por parte de los empleados, tomando en cuenta que los problemas deben someterse a la consulta y el mismo culmina con cuando entre todos llegan a una decisión final (Kinicki y Kreitner, 2003). El término toma de decisiones ha sido definido por diferentes autores, poniendo unos mayor énfasis que otros en algunas cuestiones; pero coincidiendo todos, en que se trata de una elección de una variante de conducta dentro de varias alternativas posibles para la solución de un problema dado.

El indicador solución de conflictos, cuenta con el 20\% de los trabajadores casi siempre posee competencias para la solución de conflictos laborales, $40 \%$ algunas veces, $20 \%$ pocas veces y $20 \%$ nunca. Estos resultados demuestran claramente que existe debilidades se infiere que los trabajadores tienen debilidades en cuanto al indicador solución de conflictos por lo tanto también debilidades en las relaciones interpersonales, hecho que es contrario a lo dicho por Chiavenato (2008), en donde considera que las relaciones interpersonales determina como se relacionan las personas en la organización y que grado de libertad existe en esa relación. Si las personas trabajan aisladas entre sí o en equipos de trabajos, a través de una intensa interrelación humana.

\section{Conclusiones}

Existe una debilidad en la organización tomando en cuenta que la mayoría de los trabajadores algunas veces y pocas veces poseen las habilidades y los conocimientos necesarios para el desarrollo de sus funciones como gerente educativo, además algunas veces y pocas veces toman decisiones en el desarrollo de sus funciones. Con respecto a la dimensión de motivación los 
resultados fueron similares, en el sentido de que algunas veces y pocas veces los trabajadores cuando desarrolla sus funciones motivan al resto de sus compañeros que dependen de su gerencia.

Las actividades que se lleve a cabo en la institución se aplican un liderazgo grupal, pocas veces se desarrolla él trabajo en equipo para alcanzar las metas y objetivos institucionales y algunas veces ponen en práctica la comunicación entre el gerente y el resto de los trabajadores como una herramienta que permite alcanzar la calidad del servicio prestado.

El talento humano de la organización requiere proseguir en la formación del empowerment como estrategia de empoderamiento gerencial, de ese modo, existirá mayor posibilidad de que se genere un liderazgo comprometido en alcanzar el mayor nivel de productividad en conformidad de relación con la filosofía organizacional de la empresa.

\section{Referencias}

1. Acosta, A. (2002). Empowerment. Información digital. Recuperado de http://www.improvenconsultores.com

2. Aldana, J., \& Piña, J. (2017). Calidad del servicio prestado al cliente por los instructores de gimnasios. Revista Arbitrada Interdisciplinaria Koinonía, 2(3), 172-197. Recuperado de http://fundacionkoinonia.com.ve/ojs/index.php/revistakoinonia/article/view/59/46

3. Cálix, Carlos Geovany, \& Martínez, Lisana B., \& Vigier, Hernán P., \& Núñez, José Jairo (2016). El Rol del Empowerment en el Éxito Empresarial. Recuperado de https://www.redalyc.org/pdf/4560/456046142002.pdf

4. Chiavenato, I. (2008) Administración de los Nuevos Tiempos. Editorial McGrawHill. (1era ed.). México.

5. Díaz. E. (2005) Empowerment, como herramienta en las organizaciones. Editorial. Trillas. México.

6. Johnson, Y. (2005). Empowerment. Recuperado de http://es.slideshare.net/alexander_hv/coaching-yempowerment-46472148 
7. Kinicki, A. \& Kreitner, R. (2003). Organizational behavior: concepts, problems and practices. McGraw-Hill editorial.

8. Morales, M. (2000). Organizational Development, Integral Approach. Limusa editions. Mexico.

9. Newstrom. J. (1992) What every Supervisor should Know. Editorial McGRAW- HILL INTERAMERICANO, S.A. Mexico.

10. Sabino, C. (2000). The Research Process Caracas: Panapo Editorial.

11. Valdés, E. (2005). Empowerment Digital information. Recovered from http: //www.mailxmail

@2018 por el autor. Este artículo es de acceso abierto y distribuido según los términos y condiciones de la licencia CreativeCommonsAtribución-NoComercial-Compartirlgual4.0Internacional (CCBY-NC-SA4.0)

(https://creativecommons.org/licenses/by-nc-sa/4.0/). 